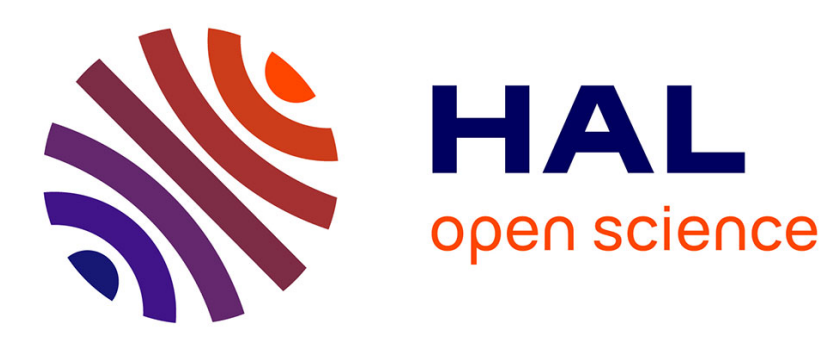

\title{
La Formation de Marsat et le Téphra CF7, marqueurs distaux d'éruptions trachytiques violentes de la Chaîne des Puys au Boréal
}

Gérard Vernet, Jean-Paul Raynal

\section{- To cite this version:}

Gérard Vernet, Jean-Paul Raynal. La Formation de Marsat et le Téphra CF7, marqueurs distaux d'éruptions trachytiques violentes de la Chaîne des Puys au Boréal. Quaternaire, 2008, 19 (2), pp.97106. halshs-00291721

\section{HAL Id: halshs-00291721 https://shs.hal.science/halshs-00291721}

Submitted on 29 Jun 2008

HAL is a multi-disciplinary open access archive for the deposit and dissemination of scientific research documents, whether they are published or not. The documents may come from teaching and research institutions in France or abroad, or from public or private research centers.
L'archive ouverte pluridisciplinaire HAL, est destinée au dépôt et à la diffusion de documents scientifiques de niveau recherche, publiés ou non, émanant des établissements d'enseignement et de recherche français ou étrangers, des laboratoires publics ou privés. 


\title{
LA FORMATION DE MARSAT ET LE TÉPHRA CF7, MARQUEURS DISTAUX D'ÉRUPTIONS TRACHYTIQUES VIOLENTES DE LA CHAÎNE DES PUYS AU BORÉAL
}

\author{
Gérard VERNET ${ }^{1} \&$ Jean-Paul RAYNAL ${ }^{2}$
}

\begin{abstract}
RÉSUMÉ
Deux marqueurs distaux d'éruptions trachytiques violentes ont été découverts en relation de contemporanéité avec des niveaux archéologiques mésolithiques. Le premier, identifié en 1995, est une coulée boueuse synéruptive, identifiée en bordure de Limagne, au sein de la Formation de Marsat, à plus de onze kilomètres de son volcan source le puy Chopine. Le second, Téphra de la Rodde ou Téphra CF7, observé clepuis 1997 sur plusieurs coupes de Limagne, présente un faciès original à fragments trachytiques anguleux centimétriques, témoins de l'explosion d'un dôme trachytique, mis en place par retombée directe ou inclus dans des faciès proximaux de coulées pyroclastiques. Au cours du Boréal, la Limagne occidentale a donc enregistré au moins deux épisodes éruptifs trachytiques violents de la Chaîne des Puys. Ces éruptions ont touché directement des occupations mésolithiques et ont affecté profondément les biotopes.
\end{abstract}

Mots-Clés : Massif central, Chaîne des Puys, trachytes, téphras, éruptions violentes, Boréal, Mésolithique, impact environnemental.

\section{ABSTRACT}

THE MARSAT FORMATION AND THE CF7 TEPHRA, DISTAL MARKERS OF VIOLENT TRACHYTIC ERUPTION OF THE CHAINE DES PUYS DURING THE BOREAL PERIOD

Two eruptive markers of trachytic geochemistry have been identified in close relationships with Mesolithic archaeological layers. A syneruptive mudflow related to the eruption of puy Chopine was identified in 1995 at Marsat, eleven kilometres from the source-volcano, on the Limagne border. Another tephra, named Tephra de la Rodde or CF7, has been observed since 1997 in several sections in the Limagne plain. It is characterized by angular centimetric fragments generated by a dome explosion, either included in pyroclastic flows or emitted as a fall. During the Boreal, two main violent trachytic eruptions of the Chaine des Puys had a direct impact upon environments and Mesolithic occupations of the Limagne.

Key-words: Massif central, Chaîne des Puys, trachyte, tephra, high explosivity eruption, Boreal, mesolithic, environmental impact.

\section{1 - INTRODUCTION}

Les éruptions des volcans de la Chaîne des Puys au cours du Tardiglaciaire et de l'Holocène ont émis des téphras à plus ou moins large dispersion (Brousse et al., 1969 ; Baudry \& Camus, 1972 ; Brousse \& Rudel, 1973). Des retombées ont ensuite été repérées dans différents environnements palustres et lacustres d'un vaste espace comprenant la Limagne occidentale et plus à l'Est les Monts du Forez et s'étendant vers le Sud à la Chaîne des Puys méridionale, aux Monts Dore, à l'Artense, au Cézallier et au Cantal (Juvigné, 1983, 1987, 1991, 1992, 1993 ; Juvigné \& Gilot, 1986 ; Juvigné \& Gewelt, 1987 ; Juvigné et al., 1987 ; Etlicher et al., 1987; Juvigné et al., 1992). Treize téphras ont alors constitué une première téphrostratigraphie de référence, avec du plus ancien au plus récent :
- Téphra de la Nugère,

- Téphra T5 de la Godivelle,

- Téphra T4 de la Godivelle,

- Téphra du Puy de Dôme,

- Téphra de la Taphanel,

- Téphra du Pariou,

- Téphra de la Vache-Lassolas,

- Téphra d'Anglard,

- Téphra du Montcineyre,

- Téphra du Pavin,

- Téphra de Beaunit,

- Téphra de Fung,

- Sables d'Espinasse.

Trois de ces téphras (Téphra de la Taphanel, Téphra du Pariou et Téphra de la Vache-Lassolas) sont situés dans le Boréal mais un seul d'entre eux est de nature

\footnotetext{
I INRAP et UMR 6042 CNRS, Université Clermont-Ferrand 2, 4 rue Ledru, 63057 Clermont-Ferrand Cedex 1 . Courriel : gerard.vernet@inrap.fr
}

${ }^{2}$ Université de Bordeaux 1, UMR 5199 PACEA, IPGQ, avenue des Facultés, bâtiment B18, 33405 TALENCE. Courriel : jp.raynal@wanadoo.fr 


\title{
LA FORMATION DE MARSAT ET LE TÉPHRA CF7, MARQUEURS DISTAUX D’ÉRUPTIONS TRACHYTIQUES VIOLENTES DE LA CHAÎNE DES PUYS AU BORÉAL
}

\author{
Gérard VERNET ${ }^{1} \&$ Jean-Paul RAYNAL ${ }^{2}$
}

\begin{abstract}
RÉSUMÉ
Deux marqueurs distaux d'éruptions trachytiques violentes ont été découverts en relation de contemporanéité avec des niveaux arrchéologiques mésolithiques. Le premier, identifié en 1995, est une coulée boueuse synéruptive, identifiée en bordure de Limagne, au sein de la Formation de Marsat, à plus de onze kilomètres de son volcan source le puy Chopine. Le second, Téphra de la Rodde ou Téphra CF7, observé depuis 1997 sur plusieurs coupes de Limagne, présente un faciès original à fragments trachytiques anguleux centimétriques, témoins de l'explosion d'un dôme trachytique, mis en place par retombée directe ou inclus dans des faciès proximaux de coulées pyroclastiques. Au cours du Boréal, la Limagne occidentale a donc enregistré au moins deux épisodes éruptifs trachytiques violents de la Chaîne des Puys. Ces éruptions ont touché directement des occupations mésolithiques et ont affecté profondément les biotopes.
\end{abstract}

Mots-Clés : Massif central, Chaîne des Puys, trachytes, téphras, éruptions violentes, Boréal, Mésolithique, impact environnemental.

\section{ABSTRACT}

THE MARSAT FORMATION AND THE CF7 TEPHRA, DISTAL MARKERS OF VIOLENT TRACHYTIC ERUPTION OF THE CHAINE DES PUYS DURING THE BOREAL PERIOD

Two eruptive markers of trachytic geochemistry have been identified in close relationships with Mesolithic archaeological layers. A syneruptive mudflow related to the eruption of puy Chopine was identified in 1995 at Marsat, eleven kilometres from the source-volcano, on the Limagne border. Another tephra, named Tephra de la Rodde or CF7, has been observed since 1997 in several sections in the Limagne plain. It is characterized by angular centimetric fragments generated by a dome explosion, either included in pyroclastic flows or emitted as a fall. During the Boreal, two main violent trachytic eruptions of the Chaîne des Puys had a direct impact upon environments and Mesolithic occupations of the Limagne.

Key-words: Massif central, Chaîne des Puys, trachyte, tephra, high explosivity eruption, Boreal, mesolithic, environmental impact.

\section{1 - INTRODUCTION}

Les éruptions des volcans de la Chaîne des Puys au cours du Tardiglaciaire et de 1'Holocène ont émis des téphras à plus ou moins large dispersion (Brousse et al., 1969 ; Baudry \& Camus, 1972 ; Brousse \& Rudel, 1973). Des retombées ont ensuite été repérées dans différents environnements palustres et lacustres d'un vaste espace comprenant la Limagne occidentale et plus à l'Est les Monts du Forez et s'étendant vers le Sud à la Chaîne des Puys méridionale, aux Monts Dore, à l'Artense, au Cézallier et au Cantal (Juvigné, 1983, 1987, 1991, 1992, 1993 ; Juvigné \& Gilot, 1986 ; Juvigné \& Gewelt, 1987 ; Juvigné et al., 1987 ; Etlicher et al., 1987; Juvigné et al., 1992). Treize téphras ont alors constitué une première téphrostratigraphie de référence, avec du plus ancien au plus récent :
- Téphra de la Nugère,

- Téphra T5 de la Godivelle,

- Téphra T4 de la Godivelle,

- Téphra du Puy de Dôme,

- Téphra de la Taphanel,

- Téphra du Pariou,

- Téphra de la Vache-Lassolas,

- Téphra d'Anglard,

- Téphra du Montcineyre,

- Téphra du Pavin,

- Téphra de Beaunit,

- Téphra de Fung,

- Sables d'Espinasse.

Trois de ces téphras (Téphra de la Taphanel, Téphra du Pariou et Téphra de la Vache-Lassolas) sont situés dans le Boréal mais un seul d'entre eux est de nature

\footnotetext{
${ }^{1}$ INRAP et UMR 6042 CNRS, Université Clermont-Ferrand 2, 4 rue Ledru, 63057 Clermont-Ferrand Cedex 1 . Courriel : gerard.vernet@inrap.fr

${ }^{2}$ Université de Bordeaux I, UMR 5199 PACEA, IPGQ, avenue des Facultés, bâtiment BI8, 33405 TALENCE. Courriel : jp.raynal@wanadoo.fr
} 


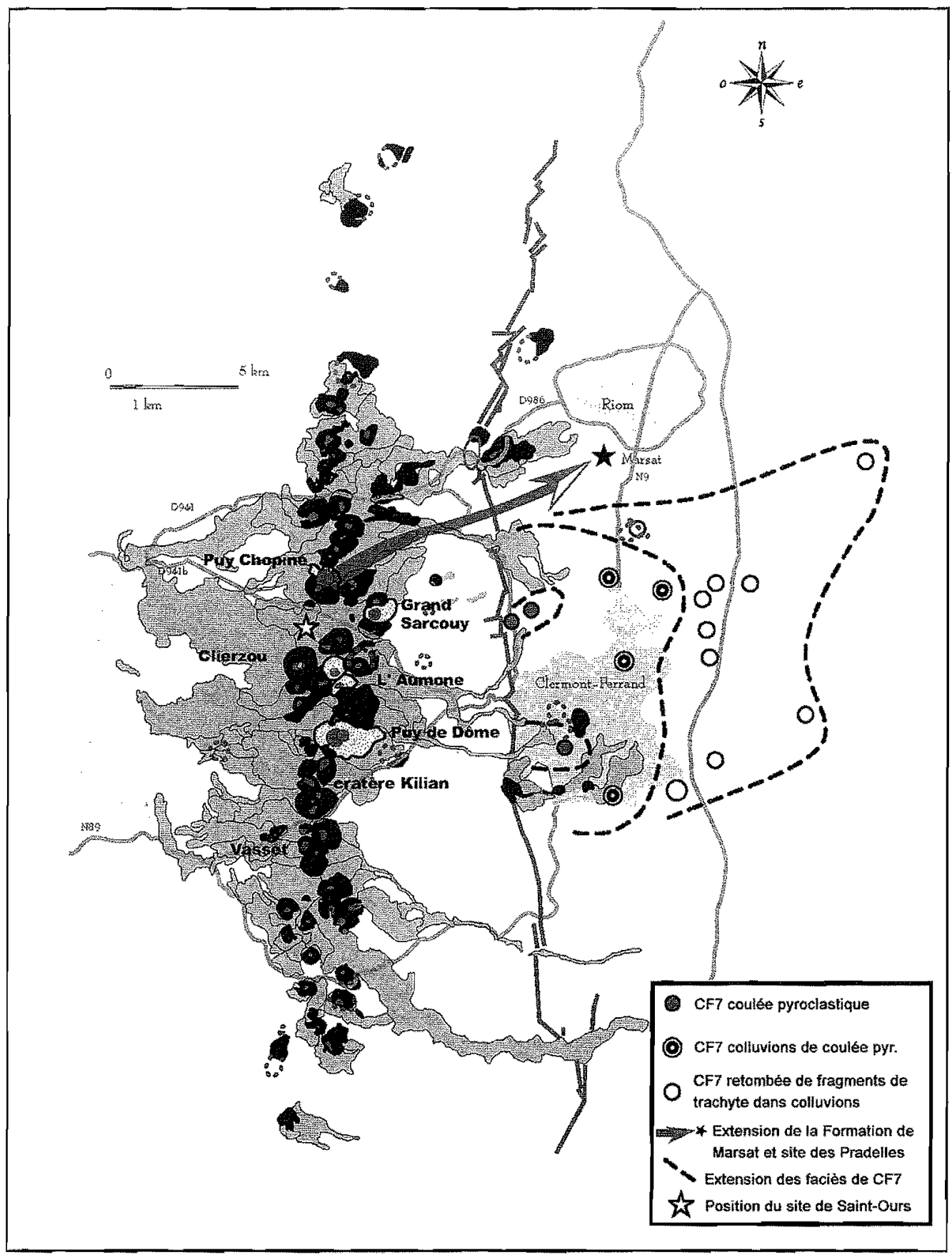

Fig. 1 : Chaîne des Puys et Limagne. Extension des formations étudiées (Formation de Marsat et CF7). Fig. 1: Map of the Chaine des Puys and the Limagne with extent of the Marsat Formation and CF7 Tephra.

trachytique : le Téphra de la Taphanel (Juvigné, 1987). Juvigné (1987, 1992 et 1993) propose que ce téphra résulte d'un mélange des produits issus de trois édifices (Chopine, Kilian et Vasset), qu'il faut admettre pour cette retombée un âge moyen d'environ $8500 \mathrm{BP}$ et que son aire de dispersion vers le Sud forme le plus vaste des lobes des téphras tardiglaciaires et holocènes de la Chaîne des Puys. Les produits trachytiques issus du Puy de Dôme (Téphra du Puy de Dôme), connus uniquement dans l'environnement méridional proche de ce volcan (Narse d'Ampoix ; Juvigné \& Gewelt, 1987) sont situés à la transition Dryas récent/Préboréal et ont été datés de 9800 BP (Juvigné \& Gewelt, 1987).
Depuis une vingtaine d'années, nous avons pour notre part étudié de façon systématique les téphras préservés au pied de la Chaîne des Puys, en Limagne d'Auvergne, où se sont multipliées les observations dans le cadre de l'Archéologie préventive durant la dernière décennie (fig. 1) (Raynal et al., 1988 ; Vernet et al., 1990 ; Vernet, 1992 ; Raynal et al., 1994 ; Vernet \& Raynal, 1995; Vernet et al., 1996 ; Vernet et al., 1998 ; Vernet \& Raynal, 2000a, 2000b ; Vernet \& Raynal, 2001a ; Vernet \& Raynal, 2002 ; Raynal et al., 2003 ; Miallier et al., 2004a ; Fourmont et al., 2006). Située immédiatement à l'Est de la zone éruptive, la Limagne occidentale recèle encore aujourd'hui de très 
nombreuses zones humides (palustres et lacustres) qui un fonctionné pendant le Tardiglaciaire et le début de I'Holocène et permis une bonne préservation des iephras dans des séquences sédimentaires relativement dilatécs et favorables à l'obtention de repères chronologiques (palynologie, radiocarbone). L'ensemble des données obtenues a débouché sur la proposition d'une tephrostratigraphie locale comportant onze téphras repérés entre 15000 et $8000 \mathrm{BP}$. Deux de ces dépôts pyroclastiques sont des marqueurs de l'activité éruptive trachytique violente de la Chaîne des Puys au Boréal qui a affecté les biotopes et touché directement des occupations mésolithiques.

\section{2 - LE CADRE \\ TÉPHROSTRATIGRAPHIQUE ÉTABLI EN LIMAGNE OCCIDENTALE}

Les onze recouvrements téphriques reconnus montrent l'importance et la fréquence des éruptions pendant le Tardiglaciaire et l'Holocène et constituent une trame téphrostratigraphique fiable, même si l'identification du volcan source n'est pas toujours résolue. Elle précise la chronologie des éruptions, complète les informations obtenues dans les autres secteurs géographiques (Juvigné, 1993) et change quelque peu la vision de l'impact des éruptions de la Chaîne des Puys sur les paléoenvironnements. Du plus ancien au plus récent, on a reconnu (fig. 2):

- La retombée de Cellule,

- Le complexe téphrique CF1a/CF1b (retombée de la rue de la Barre/retombée des Roches),
- La retombée de la Moutade,

- Le Téphra CF2 (ou retombée de la rue Descartes),

- Le Téphra CF3 (ou retombée de la rue de Montjuzet),

- Le Téphra CF4 (ou retombée ancienne de la rue de Blanzat),

- Le Téphra CF5 (ou retombée de la rue Sous-lesVignes),

- La Formation de Marsat: il s'agit d'un des deux téphras décrits ici (cf. chapitre 3),

- Le Téphra CF6 (ou retombée récente de la rue de Blanzat),

- Le Téphra CF7 (ou téphra de la Rodde) : il s'agit d'un des deux téphras décrits ici (cf. chapitre 4),

- Le Téphra de Sarliève.

Six de ces téphras (de CF3 à CF7) sont calés dans le

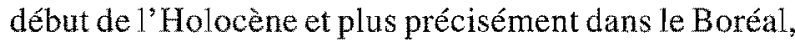
soit une période de l'ordre de 2000 ans. Trois de ces téphras (CF5, Formation de Marsat et CF7) possèdent une composition chimique qui les place dans le domaine des trachytes, voire des rhyolites. Ils correspondent au maximum de l'épisode éruptif trachytique d'âge holocène de la Chaîne des Puys, qui voit la mise en place du Puy de Dôme (durant le Préboréal), du puy Chopine, du puy Vasset et du cratère Kilian (durant le Boréal). Pendant ce même épisode bioclimatique, il ne faut pas oublier la mise en place du puy de Pariou qui présente une phase explosive à magma trachytique (Boivin et al., 2004b). Des données récentes obtenues dans la Chaîne des Puys permettent de proposer que la durée totale d'activité des trois volcans trachytiques n'a pas excédé quelques siècles autour du $10^{\circ}$ millénaire (Miallier et al., 2004b). Deux des téphras trachytiques, repérés en Limagne et calés dans le Boréal,

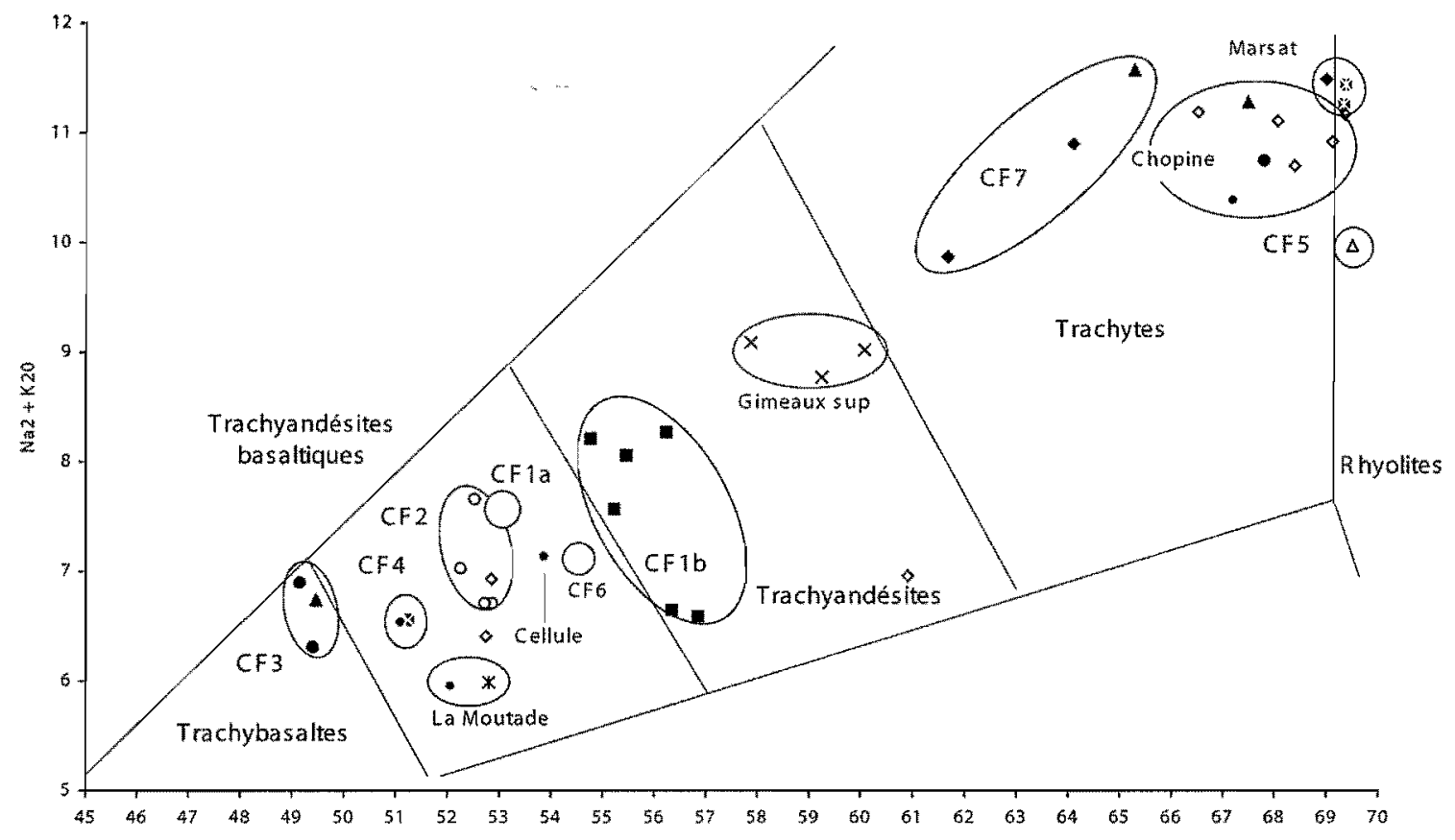

Fig. 2 : Géochimie des téphras de Limagne occidentale (analyse roche totale sur scories).

Fig. 2: Geochemistry of the different tephra beds identified in Westem Limagne (whole rock analysis on scoriae). 
présentent des faciès et des micro-faciès qui démontrent la violence des éruptions responsables de leur mise en place ; il s'agit de la formation de Marsat et du Téphra CF7.

\section{3 - LA FORMATION DE MARSAT}

La formation de Marsat a été identifiée en 1995, lors de la construction d'un ouvrage de rétention des crues,

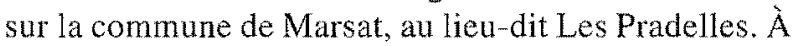
l'emplacement du site, elle surmonte deux niveaux archéologiques (niveaux 5.2 et 5.3, fig. 3). L'un des niveaux archéologiques a été fouillé sur dix mètres carrés ce qui a permis de reconnaître les restes d'un campement de chasseurs de la fin des temps glaciaires, établi sur la berge d'un cours d'eau et recouvert par les produits du Puy Chopine (Vernet \& Raynal, 2000b; Vernet et al., 2001b). Les outils de pierre taillée sont peu nombreux et fabriqués dans des silex locaux. La faune des Pradelles ne comprend pratiquement que des restes de Bos primigenius. La présence de charbons de bois et d'ossements brûlés atteste que l'on se trouve sans doute à la périphérie d'une zone d'activité. La conservation des vestiges organiques (feuilles, bois) en fait un site exceptionnel de la fin des temps glaciaires au pied des volcans (Vernet, 2004).

\section{1 - LA STRATIGRAPHIE DES DÉPÔTS PYROCLASTIQUES}

La formation de Marsat est constituée de trois unités. De bas en haut, on distingue (fig. 3 ):

- un niveau compact et homogène de couleur beige contenant de très nombreux fragments anguleux. centimétriques à pluricentimétriques, de trachyte blanc frais, et de nombreuses empreintes de végétaux, feuilles et brindilles. Localement, cette unité (niveau 4 de la figure 3) se termine par une mince lamine pouvant représenter une retombée directe de produits fins. Les fragments de trachyte présentent une composition minéralogique à sphènes. Il s'agit indiscutablement d'un trachyte de la Châ̂ne des Puys, provenant du Chopine ou du Sarcouy. L'analyse chimique $(69,36 \%$ de $\mathrm{SiO}_{2}$ ) (Le Bas et al., 1986) (fig. 2) exclut le Sarcouy et confirme le puy Chopine comme volcan émetteur. Cette première unité représente donc une manifestation de l'éruption trachytique du puy Chopine, arrivée en Limagne selon l'axe de drainage que représente le ruisseau de Mirabel, sous la forme d'une coulée boueuse synéruptive,

- une seconde unité (niveau 3 de la figure 3) repose sur la précédente par une discordance de ravinement fortement marquée sous la forme d'un chenal en U, à berges raides $\left(40^{\circ}\right.$ à $\left.55^{\circ}\right)$, d'une largeur moyenne de 7 mètres et d'une profondeur d'au moins 3 mètres. Ce chenal est comblé de matériaux très grossiers, composés quasi exclusivement de galets décimétriques de trachyte dans une matrice de graviers également de nature trachytique. Plusieurs troncs d'arbres profondément ferruginisés étaient visibles à la base de ce chenal. Cette unité représente la réorganisation du réseau de drainage après l'invasion de la paléo-vallée du ruisseau de Mirabel par les produits du début de l'éruption trachytique du Puy Chopine. Ce phénomène est synéruptif.

- la troisième unité (niveau 2 de la figure 3) est composée de matériel fin trachytique $\left(68,99 \%\right.$ de $\left.\mathrm{SiO}_{2}\right)$ (fig. 2), organisé en fines lamines qui représentent des retombées de panache éruptif vannées.

Ces trois unités, qui constituent la formation de Marsat, représentent donc un détail nouveau dans les processus éruptifs du puy Chopine, en position distale orientale, différent de celui des nuées pyroclastiques habituellement rencontrées sur le plateau des Dômes, en position plus proximale. Les produits trachytiques présents à Marsat-Les-Pradelles proviennent sans ambiguité du puy Chopine. Leur composition chimique est très proche de celle de la lave de l'aiguille terminale de cet édifice, mais également de celle des fragments de lave juvénile du premier recouvrement des phases éruptives de ce même édifice identifié à Saint-Ours (Vernet et al., 2001b).

La datation de la mise en place de la formation de Marsat est assurée par une nouvelle datation $14 \mathrm{C}$ de $8465+$ ou -70 BP (ARD 1516) obtenue sur des charbons de bois provenant de la base des produits proximaux du Puy Chopine à Saint-Ours-les-Roches : elle confirme les âges précédemment obtenus pour cette

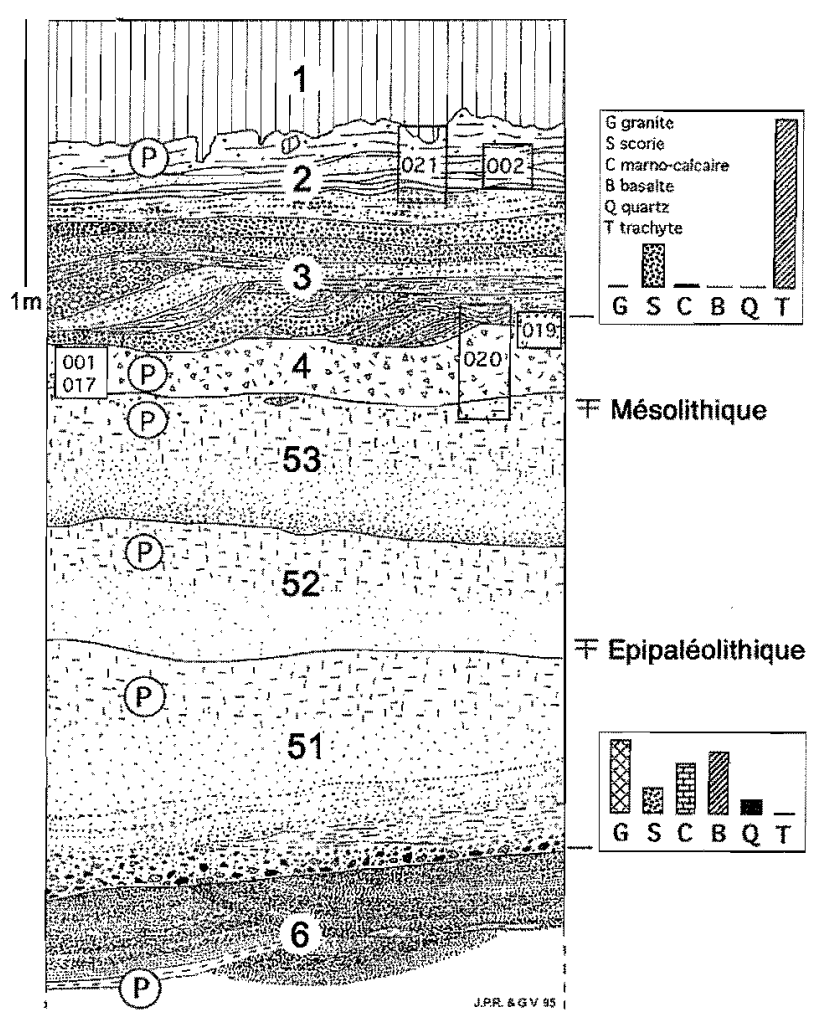

Fig. 3 : Coupe stratigraphique du site de Marsat. Niveaux 4,3 et $2=$ Formation de Marsat.

Fig. 3: Stratigraphy at Marsat prehistoric site. Units 4,3 and 2 constitute the Marsat Formation. 
aruption co fixe également un âge minimum pour les mivatux archeologiques sous-jacents.

\subsection{I.ES FACIÈS DE LA FORMATION DE MARSAT, TIMOINS DE LA VIOLENCE DE L'ÉRUPTION DU PUY CHOPINE}

Le faciès de l'unité de base de la formation de Marsat caractérise une coulée boueuse synéruptive, identifiée à plus de $11 \mathrm{~km}$ de son volcan-source. Les études réalisćes sur le site de Marsat et sur les produits proximaux de l'éruption du puy Chopine à Saint-Ours (Vernet $e t$ al., 200 (b) nous permettent de proposer que la coulée boueuse de Marsat corresponde à un faciès distal d'une des coulées pyroclastiques observées en position proximale. Nous avons pu observer le changement de nature d'une même formation (passage progressif d'une coulée pyroclastique à une coulée boueuse) sur des volcans italiens comme le Vésuve et le Vulture (Vernet et al., 1999). Cette variation latérale de faciès esı due en particulier à la diminution de la température, le passage de l'eau de l'état gazeux à l'état liquide. Dans les produits issus du puy Chopine, on note la présence de nombreux fragments calcinés de branches en position proximale (à Saint-Ours), alors qu'en position distale (à Marsat) les fragments de végétaux (branches et feuilles) ne sont pas calcinés (pas de conservation, exclusivement des empreintes). L'absence de vésicules dans les sédiments de la coulée de Marsat montre qu'il ne restait plus d'eau à l'état gazeux lors du dépôt du matériel pyroclastique. On note l'absence de gros éléments charriés dans le corps de la coulée ce qui traduit la faible compétence de celle-ci lors de son arrivée dans la vallée du ruisseau de Mirabel. En revanche, à SaintOurs, la présence de fragments de troncs, de branches et de fragments d'écorces met en évidence une destruc tion importante du couvert végétal sur le plateau des Dômes. C'est pour le moment le seul exemple de coulée boueuse décrite avec une extension aussi importante pour un édifice de la Chaîne des Puys et mise en corrélation avec des produits de coulées pyroclastiques présents en position proximale.

Le faciès de la seconde unité de la formation de Marsat permet de la rattacher à un dépôt de type torrentiel. Il résulte d'un phénomène de type catastrophique visant à rétablir le drainage dans la vallée du ruisseau de Mirabel qui avait été fortement perturbé par la mise en place de la coulée boueuse. Ces écoulements torrentiels ont été violents. Le ravinement a été intense avec la création de chenaux de plusieurs mètres de profondeur. Ils ont également détruit la végétation et en particulier les arbres présents dans la vallée; on note la présence de nombreux troncs charriés dans les chenaux (diamètres des troncs observés de l'ordre de 0,50 à $0,60 \mathrm{~m}$ )

La troisième unité de la formation de Marsat montre que l'éruption du puy Chopine a également engendré la formation de panaches éruptifs de cendres qui se sont développés vers l'Est. L'épaisseur de ces dépôts cendreux (en moyenne $0,30 \mathrm{~m}$ ) permet de calculer que la
Limagne dans la zone de Riom a dû recevoir environ 3000 mètres cubes de cendres trachytiques à l'hectare ce qui n'est pas négligeable et a forcément perturbé les paléoenvironnements. Une perturbation des cortèges palynologiques a été démontrée pour des recouvrements estimés plus réduits, par exemple pour le téphra CF5 (Vivent \& Vernet, 2001).

L'importance et la nature des dépôts éruptifs du puy Chopine découverts à Marsat changent de façon radicale la vision que les volcanologues avaient jusqu'à présent des éruptions trachytiques de la Chaîne des Puys : nul n'imaginait qu'une coulée boueuse synéruptive ait affecté la Limagne (fig. 1), vers 8500 ans BP. L'impact de l'éruption du puy Chopine sur les environnements en position distale est ici particulièrement démonstratif. En effet, la coulée boueuse ennoie et colmate la vallée du ruisseau de Mirabel à son débouché sur la plaine de la Limagne. Elle fossilise au passage un campement mésolithique établi en bordure du ruisseau. Le drainage naturel est alors totalement perturbé. La réorganisation du réseau de drainage intervient de façon catastrophique par la création de chenaux comblés d'alluvions torrentielles (composées exclusivement de blocs et galets de trachyte) qui charrient des troncs d'arbres, témoins de la destruction de la végétation par les phénomènes éruptifs. Enfin, la vallée est recouverte par au moins trente centimètres de cendres de retombées de panache éruptifs vannées. Ce dernier événement perturbe encore le drainage de cette vallée et plus largement la végétation de la Limagne. Il est clair que ce secteur de plaine se trouve alors temporairement dégradé, voire inhospitalier. Il faut garder à l'esprit que la même éruption a anéanti le couvert végétal sur le plateau des dômes sur une surface importante centrée sur le puy Chopine (cf. recouvrement trachytique du puy Chopine sur la carte de la Chaîne des Puys, Boivin et al., 2004a). Ces recouvrements proximaux (dépôts de déferlantes basales) sont constitués d'au moins quatre nappes de recouvrement (Vernet et al., 2001b) :

- Nappe 1 : activité explosive à cheminée ouverte (activité vulcanienne),

- Nappe 2 : explosion nettement phréatomagmatique,

- Nappe 3: explosion phréatomagmatique moins violente,

- Nappe 4: produits résultant d'un écoulement sous forme plus ou moins boueuse.

Ces nappes sont scellées par un dépôt de fragments trachytiques «moussus » qui évoque une retombée plinienne.

En résumé, l'éruption du puy Chopine a donc engendré des écoulements qui ont atteint la Limagne (Formation de Marsat) au Nord-Est de l'édifice et dont l'origine est à rechercher dans les nappes de recouvre-ment 1 et 4 décrites en position proximale.

La mise en évidence d'une retombée plinienne à Saint-Ours au Sud de l'édifice confirme le caractère violent de cette éruption et nous proposons de rattacher à ce panache éruptif plinien le Tephra de la Taphanel 
(Juvigné, 1987), le plus largement dispersé vers le sud parmi les téphras tardiglaciaires et holocènes de la Chẫne des Puys (Juvigné, 1993).

\section{4 - LE TÉPHRA CF7 (OU TÉPHRA DE LA RODDE)}

Depuis 1997, de nombreuses coupes observées au nord-est, à l'est et au sud de Clermont-Ferrand, ont montré la présence de nodules de trachyte, soit dispersés dans des colluvions (niveau limoneux beige ou niveau argileux de type « terres noires » de Limagne), soit associés à un niveau compact cendro-limoneux beige orangé. Ces éléments sont toujours en position stratigraphique terminale des retombées connues en Limagne et surmontent en particulier CF6 (attribué au Pariou) et CF5 (attribué au cratère Kilian ou au puy Vasset). Cette position stratigraphique nous conduit, en première analyse, à proposer un âge autour de 9500 ans pour le dépôt de ces éléments trachytiques (Vernet \& Raynal, 2002). Signalons ici que sur le plateau des Dômes, au pied du Pariou et sur sa grande coulée orientale, un dépôt de déferlante contenant des éléments trachytiques de même nature que ceux qui sont observés en Limagne, a été mis en évidence (Miallier et al., 2004a), ce qui confirme donc un âge autour de 9300 ans et une antériorité de l'éruption du Pariou par rapport au dépôt de ces éléments trachytiques (CF7 ou téphra de la Rodde). Le dépôt de déferlante observé sur la coulée du Pariou est attribué au cratère Kilian et l'analyse des charbons de bois découverts à sa base donne une date de $8815 \pm 65$ (LY-12117) (Miallier et al., 2004b). En 2001, le téphra CF7 à été mis en évidence lors de la fouille d'un site mésolithique an lieudit Les Paturaux (Saintot et al., 2004), dans la banlieue est de Clermont-Ferrand. Ce téphra est indiscutablement associé au niveau archéologique et au paléosol présent en position latérale par rapport au campement mésolithique (occupation attribuée au Sauveterrien moyen). Une datation radiocarbone a été obtenue pour cette occupation humaine : $7685 \pm 145 \mathrm{BP}(7030$ à 6231 av. J.-C.) (Ly-10897). Des ossements, récoltés en 1987 dans le même secteur (La Poudrière) au niveau d'un paléosol brun, ont donné une date de $8600 \pm$ $60 \mathrm{BP}$ (7 397 à $7582 \mathrm{BC}$ ) (Ly 1687-GrA20106). Il est fort probable que ce paléosol correspond à celui qui a été observé latéralement à l'occupation mésolithique sur le site des Paturaux. Enfin, le téphra CF7 a été reconnu lors de sondages archéologiques sur le plateau des Dômes (site «Les Prés Longs » sur la commune de Pulvérières) où il est dispersé dans des limons sableux de couleur brune à brun orangé. Ces sédiments contenaient des artefacts (industrie lithique, os, céramique, coquilles de noisettes brûlées) montrant une fréquentation du site du Mésolithique au Néolithique. L'industrie lithique permet d'attribuer l'occupation mésolithique au Sauveterrien. Trois dates ont été obtenues sur les coquilles de noisettes : $9160 \pm 40 \mathrm{BP}(8460$ à $8270 \mathrm{BC}), 7610 \pm 40 \mathrm{BP}(6480$ à $6410 \mathrm{BC})$ et $6670 \pm$ $50 \mathrm{BP}$ (5660 à 5500 BC) (Colonge, 2005). De la découverte de Pulvérières, nous retiendrons que le dépôt du téphra CF7 est une nouvelle fois contemporain d'une occupation préhistorique rattachée au Sauveterrien et située au cours du $7^{\text {ème }}$ millénaire avant J.-C.

\section{1 - LES CARACTÉRISTIQUES DES FRAGMENTS LAVIQUES DE CF7}

Les fragments de lave juvénile de CF7 (fig. 4) sont de deux types:

- des fragments anguleux, gris clair à gris foncé, de lave à phénocristaux d'amphibole et de feldspaths. La vésiculation de ces fragments est réduite à très réduite (lave compacte de densité supérieure à 2). Leur morphologie suggère qu'ils proviennent de la croûte d'un dôme. Les analyses géochimiques montrent une composition dans le champ des trachytes (la teneur moyenne en silice étant de l'ordre de $64 \%$ ),

- des fragments plus arrondis, gris clair à gris beige, de lave plus pauvre en phénocristaux d'amphibole et de feldspaths. La vésiculation est importante jusqu'à
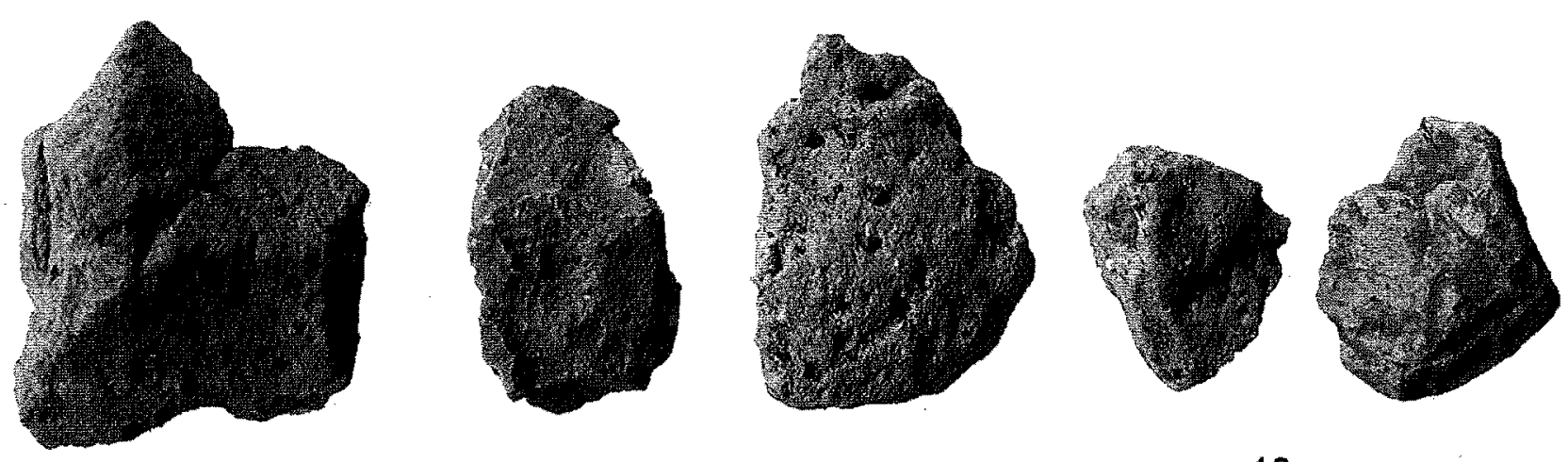

$10 \mathrm{~cm}$

Fig. 4 : Fragments de trachyte du stratotype de CF7 à la Rodde (Cébazat).

Fig. 4: Trachyte fragments from the CF7 stratotype at la Rodde (Cébazat). 
domner un uspect moussu (la densité est inférieure à 1.8). Collain éléments peuvent être considérés comme des ponces. Les analyses géochimiques montrent une conupustion dans le champ des trachytes (la teneur muycume en silice étant légèrement plus faible que celle des fragments anguleux, de l'ordre de $61 \%$; cf. Tabl. I in Miallier et al., 2004).

\section{2 - LES DIFFERENTS FACIES DE CF7}

1: observation des dépôts englobant le Téphra CF7 (ou Téphra de la Rodde), à l'cil nu et sous le microscope, permet de distinguer trois faciès différents.

1.e premier type de faciès a été observé sur des coupes dans le secteur de Nohanent (fig. 5) et dans des excavations à Chamalières. Sur ces sites, CF7 se présente sous la forme d'un niveau compact beige orangé riche en fragments laviques trachytiques de taille centimétrique (le plus gros fragment atteint une taille de $8 \mathrm{~cm}$ a Nohanent). Du point de vue granulométrique, on note une composante limoneuse importante qui lui

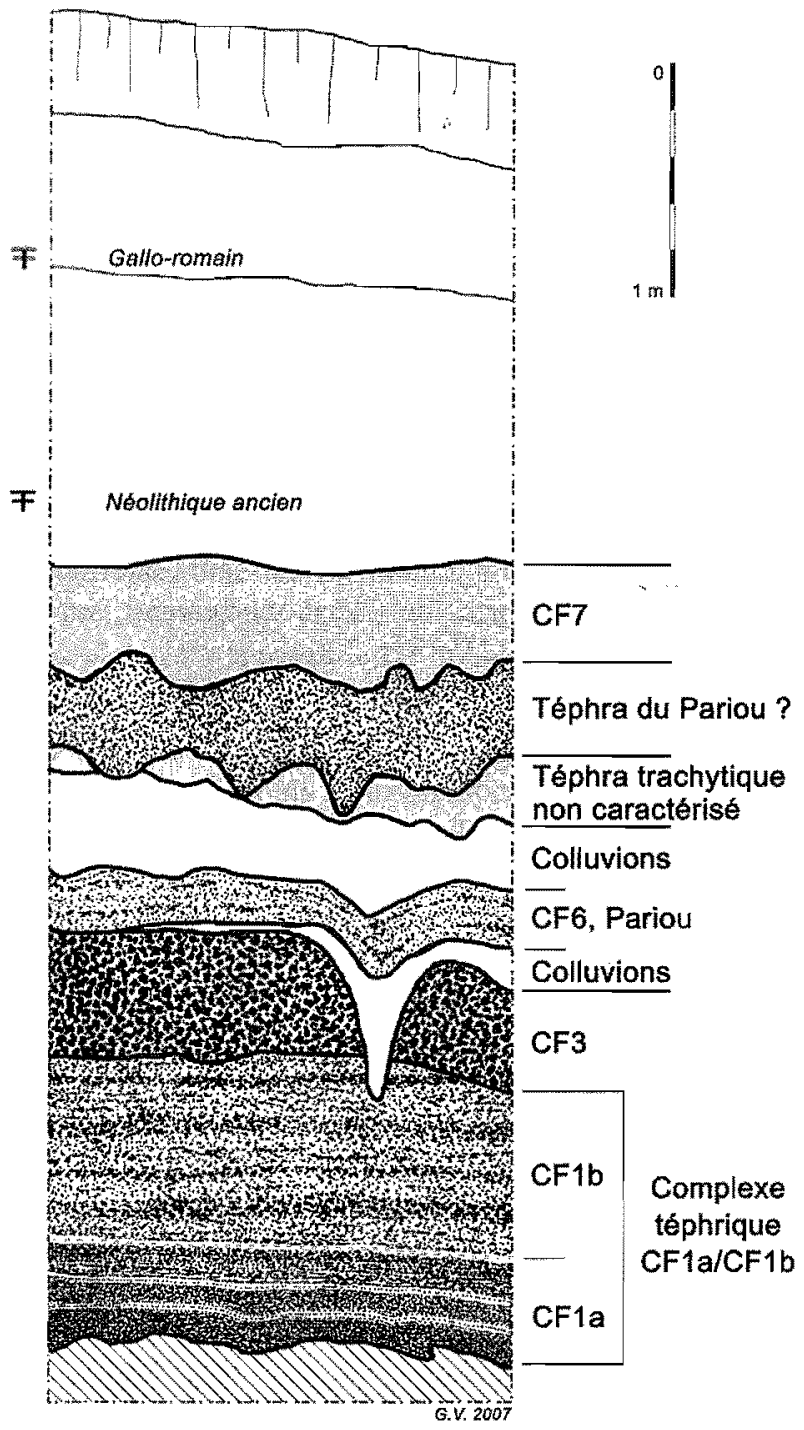

Fig. 5 : Coupe stratigraphique du site de Nohanent. Fig. 5: Stratigraphic section at Nohanent. donne un aspect «cendreux $»$ Lorsqu'il est sec. Sa puissance est de l'ordre de $0,50 \mathrm{~m}$ et sur une coupe du secteur de Nohanent, il comble un chenal et atteint une épaisseur de plus de trois mètres. Il ne présente ni litage, ni granoclassement. Ce faciès de dépôt compact, qui a tendance à s'accumuler dans les points bas, se rapproche d'un faciès de coulée pyroclastique (ou coulée boueuse) plutôt que d'une retombée. Les deux zones où l'on retrouve ce faciès de CF7 sont localisées au débouché de ravins qui entaillent l'abrupt de la faille bordière de la Limagne (cf. carte, fig. 1).

L'observation sous le microscope (fig. 6) confirme la présence de nombreux fragments plus ou moins anguleux de lave trachytique à amphiboles et feldspaths alcalins (orthoclases) dans une matrice fine beige riche en micro-fragments de lave trachytique (parfois il s'agit simplement d'une baguette de feldspath entourée d'une gangue vitreuse), accompagnés de xénoclastes essentiellement volcaniques (basalte, trachybasalte), dans une microstructure caractéristique. La matrice fine a en effet tendance à former une pellicule plus au moins épaisse autour des éléments les plus gros (fragments trachytiques et xénoclastes), oblitérant les irrégularités de leur surface et formant des éléments arrondis cendreux avec un nucleus solide : il s'agit là d'un début d'accrétion de cendres fines autour d'un germe central. Cette microstructure de proto-lapilli accrétionnés a été décrite dans des coulées pyroclastiques distales du volcan Vulture (Vernet et al., 1999). Les lapilli accrétionnés (Moore \& Peck, 1962),

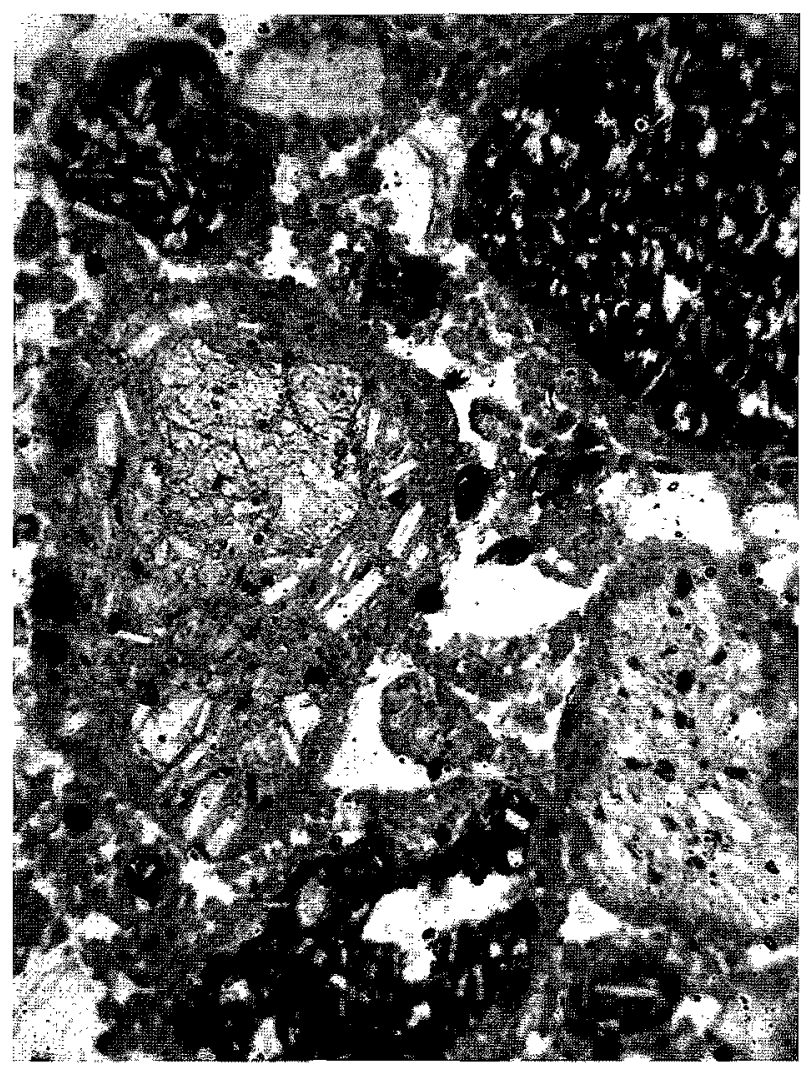

Fig. 6 : Proto-lapilli dans CF7 (Nohanent). LN, x10, le champ de la photo fait 5 mm de large.

Fig. 6: Proto-lapilli in CF7 at Nohanent, natural light, $x 10$, field of the photograph is $5 \mathrm{~mm}$ wide. 
généralement formés dans les colonnes éruptives des éruptions phréatomagmatiques, sont également connus dans les déferlantes et les coulées pyroclastiques (Walker, 1971 ; Fischer \& Schmincke, 1984 ; Cas \& Wright, 1992). Les lapilli accrétionnés in situ des surges et cosurges sont donc de bons indicateurs de dynamisme de mise en place et témoignent du régime turbulent de l'écoulement. La présence de proto-lapilli accrétionnés dans le dépôt de CF7 à Nohanent confirme son caractère de coulée pyroclastique. En outre, des micro-charbons de bois sont inclus dans la matrice cendreuse : des charbons de bois sont également présents dans les dépôts de déferlante du cratère Kilian et du Puy Chopine sur le plateau des Dômes, alors que les retombées, "dépôts froids", ne contiennent jamais de fragment ligneux. Nous sommes donc bien en présence d'une coulée pyroclatique qui a atteint la Limagne en s'écoulant par un des ravins entaillant l'abrupt de la faille bordière occidentale. Ceci renforce les observations faites à Marsat et confirme que des phénomènes violents ont accompagné les éruptions trachytiques et atteint la Limagne à plusieurs kilomètres du point éruptif.

Le deuxième type de faciès de $\mathrm{CF} 7$ a été observé dans de nombreuses coupes du bassin clermontois. Le site éponyme de La Rodde, sur la commune de Cébazat, en fournit un bon exemple (fig. 7). Un niveau plus ou moins épais (de 0,20 à $1 \mathrm{~m}$ ) de colluvions limoneuses non compactes, dont la couleur varie du beige au marron, inclut des fragments trachytiques plus ou moins anguleux, dispersés dans la masse. L'observation sous le microscope confirme la nature colluviale de cette formation et la présence de nombreuses bioturbations.

$s$

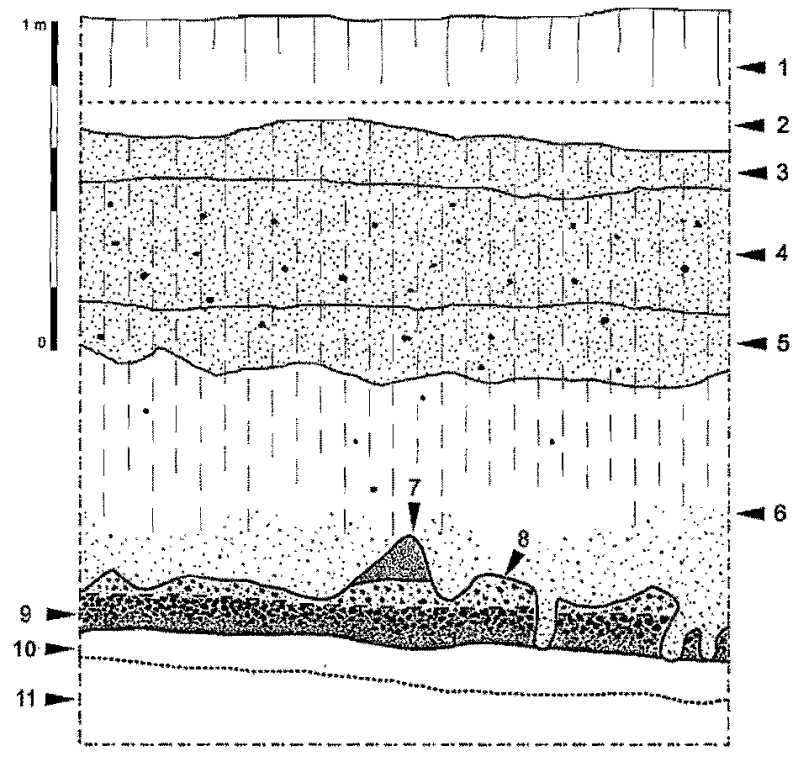

Fig. 7 : CF7 dans des colluvions limoneuses (niveaux 4 et 5) sur le stratotype de la Rodde à Cébazat.

4 et 5 , colluvions beiges à fragments de trachyte (CF7); 7, Téphra CF6, puy de Pariou ; 9, Téphra CF3, Boréal.

Fig. 7: CF7 in silty colluyiums (units 4 and 5) in the stratotype of la Rodde at Cebazat.

4 and 5, colluviums with trachyte fragments (CF7); 7, CF6 Tephra attributed to the pty de Pariou; 9, CF 3 Tephra of Boreal age.
Les éléments trachytiques sont rares à l'échelle de la lame mince. Il s'agit ici d'un téphra remanié dont le faciès d'origine peut être une coulée pyroclastique, une retombée, ou encore un mélange des deux.

Le troisième type de faciès de CF7 a été observé dans plusieurs coupes obtenues dans les formations palustres à lacustres du grand marais de Limagne. CF7 forme un lit décimétrique de fragments trachytiques plus ou moins anguleux (fig. 8). Les limites supérieures

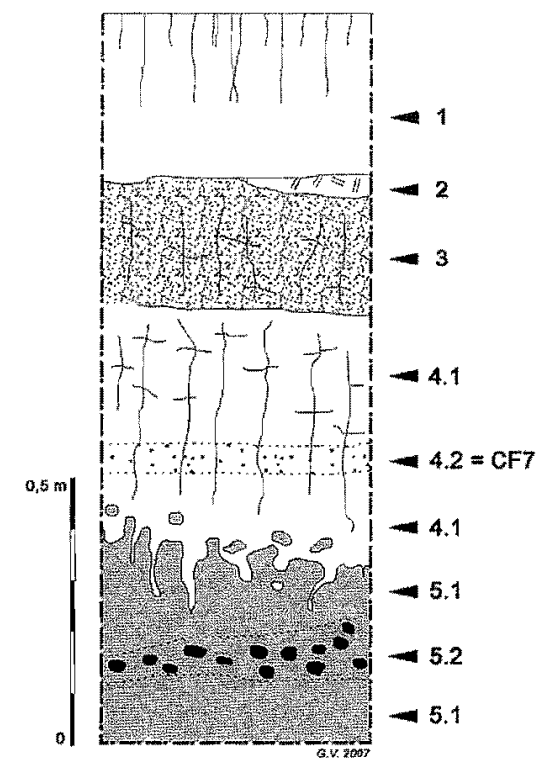

Fig. 8 : Stratigraphie du site de La Buyre à Chappes. Fig. 8: Stratigraphy of La Buyre site at Chappes.

et inférieures de ce lit sont diffuses et la matrice est de même nature que les niveaux qui l'encadrent, terre noire de Limagne ou argiles organiques palustres par exemple. Cette disposition caractérise une retombée issue d'une colonne éruptive synchrone de coulées pyroclastiques. Cette pluie de petits fragments de trachyte a touché un campement de chasseurs-cueilleurs sauveterriens aux portes de Clermont-Ferrand, sur le site « Des Paturaux » (Vernet, 2004).

\section{5 - CONCLUSIONS}

La phase éruptive à magma acide (trachy-rhyolitique) du début de l'Holocène a donné naissance aux volcans à dômes (ou protrusions) qui marquent le paysage de la Chaîne des Puys. Si le plus imposant - le Puy de Dôme - n'a pas laissé de témoin de son éruption en Limagne, d'autres édifices appartenant à cette «phase trachytique » ont en revanche émis des produits retrouvés en position distale; Téphra CF5, formation de Marsat, Téphra CF7 et Téphra de Sarliève. L'étude de deux d'entre eux, la Formation de Marsat et CF7, montre que les éruptions trachytiques ont été beaucoup plus violentes qu'on ne le pensait auparavant et qu'elles ont perturbé de façon non négligeable les environnements du plateau des Dômes et de la Limagne. Les campements des chasseurs-cueilleurs mésolithiques ont été directement affectés par les produits de ces deux éruptions. 
Ia formation de Marsat montre que l'éruption traWylique du Puy Chopine a été assez violente pour donner naissance à une coulée boueuse qui a atteint la I imagne à I I km du point éruptif (figure 1). L'impact distal de cette éruption sur les environnements est significatif. Son panache éruptif important était orienté vers le Sud : le téphra de la Taphanel (Juvigné, 1987) chl résulte et forme le lobe de dispersion le plus important reconnu en Auvergne.

Le Téphra de la Rodde (ou Téphra CF7) est composé de fragments anguleux centimétriques de trachytes, túmoins distaux de l'explosion d'un dôme. Ils sont présents sous forme de retombée dans des colluvions ou à la base des terres noires de Limagne, mais également dans des faciès proximaux de coulées pyroclastiques trachytiques près de la faille bordière occidentale de la Limagne (fig. 1). Dans les stratigraphies de Limagne, on rencontre sur la même coupe CF5, CF6 (Pariou) et CF7, du plus ancien au plus recent; toutefois, sur la coupe de Nohanent, deux téphras sont rapportables au Pariou et encadrent une retombée trachytique qui reste à caractériser précisément. Les dernières données sur la chronologie Vasset-Kilian-Pariou-Chopine (Miallier et al., 2004b) nous conduisent à proposer de rapporter CF5 à l'éruption du puy Vasset et CF7 à celle du Kilian, En Limagne et dans la cuvette clermontoise, la succession stratigraphique du plus ancien au plus récent serait donc : CF5 (Vasset) puis CF6 (Pariou) et ensuite CF7 (Kilian). Ceci permet d'apporter des précisions concernant le «téphra de Gerzat-E » (Juvigné et al., 1992), considéré comme le mélange des produits du Vasset, du Kilian et du Chopine et situé sous le « téphra de Gerzat-F » - notre Téphra CF6 - et attribué au Pariou). Il est clair désormais que le « téphra de Gerzat-E » correspond au Téphra CF5 attribué au seul volcan Vasset, puisque les produits du Kilian sont nettement dissociés et forment le faciès de retombée de CF7. Les produits indiscutablement attribués au puy Chopine et reconnus en Limagne sont uniquement ceux appartenant à la Formation de Marsat.

Au cours du Boréal, la Limagne occidentale a donc enregistré au moins deux épisodes éruptifs trachytiques violents de la Chaîne des Puys. Dans les deux cas, il a été possible d'identifier des dépôts résultant de coulées pyroclastiques en position distale ou de coulées boueuses. Ces phénomènes sont beaucoup plus dévastateurs qu'une retombée de cendres ou un épanchement de lave. Dans le cas de la formation de Marsat, un phénomène (écoulements torrentiels) de type catastrophique, visant à rếtablir le drainage dans la vallée, accentue encore le caractère dévastateur de l'éruption du Puy Chopine. Ces éruptions ont eu lieu alors que l'homme était présent et chaque fois des campements préhistoriques ont été atteints: destruction par la coulée boueuse dans le cas du site de Marsat ou simple saupoudrage par CF7 dans le cas du site « Des Paturaux ». Ces éruptions ont donc affecté les biotopes de manière significative et d'autres exemples viendront sans nul doute le confirmer.

\section{RÉFÉRENCES BIBLIOGRAPHIQUES}

BOIVIN P., BESSON J.-C., BRIOT D., CAMUS G., GOËR de HERVE A. de, GOURGAUD A., LABAZUY P., LAROUZIÉRE F.-D. de, LIVET M., MERGOIL J., MIALLIER D., MOREL J.-M., VERNET G., \& VINCENT P. M., 2004 - Carte Volcanologique de la Chatne des Puys Massif central Frangais au $1 / 25000$. $4^{\text {ene }}$ édition, Editée et réalisée par le Parc Naturel Régional des Volans d'Auvergne, Château de Montlosier, Randanne, 63970 Aydat, $120 \times 90 \mathrm{~cm}$.

BOIVIN P., BESSON J.-C., BRIOT D., CAMUS G., GOËR de HERVE A. de, GOURGAUD A., LABAZUY P., LAROUZIERE F.-D. de, LIVET M., MERGOIL J., MIALIIER D., MOREL J.-M., VERNET G., \& VINCENT P. M., 2004 - Volcanologie de la Chaine des Pays Massif Central Français, $4^{\text {eme }}$ édition, Edité par le Pare Naturel Régional des Volcans d'Auvergne, Château de Montlosier, Randanne, 63970 Aydat, (avec la participation de Jannot S. et Raynal J.-P.), $180 \mathrm{p}$.

BAUDRY D., \& CAMUS G., 1972 - Les projections volcaniques de la Chaîne des Puys et leurs utilisations. Bulletin du B.R.G.M., II (2) $, 2,1-52$

BROUSSE R., \& RUDEL A., 1973 - Activité paroxysmale de la Chaîne des Puys à 10000 ans. Bulletin historique et scientifique de l'Auvergne, t. LXXXVI, 636, 1-11.

BROUSSE R., DELIBRIAS G., LABEYRIE J., \& RUDEL A., 1969 - Eléments de chronologie des éruptions de la Chaîne des Puys. Bulletin de la Société Géologique de France, 7ème série, XI, 770-793.

CAS R.A.F., \& WRIGHT J.V., 1992 - Volcanic succesions. Modern and ancient. Chapman \& Hall ed, London, $528 \mathrm{p}$.

COLONGE D. (dir), 2005 - Rapport final d'opération de sondages et évatuations archéologiques. A89-Section 8. Le Sancy - Combronde (Pwy-de-Dôme). 2 Tomes, INRAP, février 2005, ClemontFerrand.

ETLICHER B., JANSSEN C., JUVIGNÉ E., \& Van LEEUWEN J.F.N., 1987 - Le Haut Forez (Massif central, France) après le Pléniglaciaire würmien : environnement et téphra du volcan de la Nugère. Bulletin de l'Association Française pour l'Etude du Qutaternaire, 4, 229-239.

FISCHER R.V., \& SCHMINCKE H.U., 1984 - Pyroclastic Rocks. Springer ed., Berlin, $472 \mathrm{p}$.

FOURMONT A., MACAIRE J.-J., BREHERET J.-G., ARGANT J., PRAT B., \& VERNET G., 2006 - Tephras in lacustrine sediments of the Sarliève marsh (French Massif Central) : age and preservation. Comptes-Rendus Géoscience, 338, 1141-1149.

JUVIGNÉ E., 1983 - Un marqueur stratigraphique supplémentaire dans les tourbières du Cantal : la retombee volcanique de la Taphanel. Bulletin de l'Association Française pour l'Etude du Quaternaire, 13, 3-7.

JUVIGNÉ E., 1987 - Un marqueur stratigraphique à large dispersion dans le Massif central français : la retombée du volcan Chopine vieille d'environ 8500 ans B.P. Comptes-Rendus de l'Académie des Sciences, Paris, 304 (II), $\mathrm{n}^{0} 4,187-190$.

JUVIGNÉ E., 1991 - Distribution de vastes retombées volcaniques originaires de l'Eifel et du Massif Central aux temps post-glaciaires dans le NE de la France et les régions voisines. Comptes-Rendus de l'Academie des Sciences, Paris, 312 (II), 415-420.

JUVIGNÉ E., 1992 - Distribution of widespread Late Glacial and Holocene tephra beds in the French Central Massif. Quaternary international, 13/14, 180-185.

JUVIGNÉ E., 1993 - Contribution à la téphrostratigraphie du Quaternaire et son application à la géonorphologie. Mémoire pour servir à l'Explication des Cartes Géologiques et Miniêres de la Belgique, $\mathrm{n}^{\circ} 36,66 \mathrm{p}$.

JUVIGNÉ E., \& GEWELT M., 1987 - La Narse d'Ampoix comme téphrostratotype dans la Chaîne des Puys méridionale (France), Bulletin de l'Association Française pour l'Etude du Quaternaire, 1, 37.49.

JUVIGNÉ E., \& GILOT E., 1986 - Ages et zones de dispersion des téphras émis par les volcans du Montcineyre et du lac Pavin (Massif Central, France), Zeitschrift der Deutschen Geologischen Gesellschaft, 137, 613-623, 
JUVIGNÉ E., LOUSBERG N., \& GEWELT M., 1987 - Evolution morphosedimentaire de la Narse d'Espinasse. Revue des Sciences naturelles de l'Awergne, 53, 7-14.

JUVIGNÊ E., KROONENBERG S.B., WELDKAMP A., EL ARABI A., \& VERNET G., 1992 - Widespread Allered and Boreal trachyandesitic to trachytic tephra layers as stratigraphical markers in the Massif Central, France. Quaternate, $3(344), 137$ 146.

LE BAS M.J., LE MAITRE R.W., STRECKEISEN A., \& ZANETTIN B., 1986 - A chemical classification of volcanic rocks based on the total alkali-silica diagram. Joumal of Petrology, $27,745-750$

MIALlier D., SANZELle S., PILleyre T., VERNET G. BRUGIERE S., \& DANHARA T., 2004a - Nouvelles données sur le téphra de Sarliève et le téphra CF7, marqueurs chrono-stratigraphiques de Grande Limagne (Massif central, France). ComptesRendus Géoscience, 336, 1-8.

MIALliER D., MICHON L., EVIN J., PILleYRE T., SANZELLE S., \& VERNET G., 2004b - Volcans de la Chaîne des Puys (Massif central, France) : point sur la chronologie Vasset-Kilian-Parion-Chopine. Comptes-Rendus Géoscience, 336, 1345-1353.

MOORE J.G., \& PECK D.L., 1962 - Accretionnary lapilli in volcanic rocks of the Western United States. Journal of Geology, 70 182-193

RAYNAL J.-P., MIALLIER D., VERNET G., FAIN J., CAMUS G., MONTRET M., DAUGAS J.-P., \& SANZELLE S., 1989 . Extension de la datation par thermoluminescence à une retombée trachy-andésitique rapportée au Puy de Pariou (Chaîne des Puys, France). Comptes-Rendus de l'Académie des Sciences, Paris, 308 (II), $1547-1552$

RAYNAL J.-P., VERNET J.-P., FAIN J., MIALLIER D., MONTRET M., PILLEYRE T., SANZELLE S., \& DAUGAS J.-P., 1994 - Téphrostratigraphie et préhistoire des 160 derniers millénaires en Limagne d'Auvergne (Massif central, France). Bulletin de la Société Préhistorique Française, 91 (2), 149-157.

RAYNAL J.-P., VERNET G., \& DAUGAS J.-P., 2003 - Evolution récente de la Limagne d'Auvergne (France) : impacts du voleanisme et aspects des peuplements humains au Tardiglaciaire et à l'Holocène In Climatic-envirotrmental variations and impact on Man in the circum-mediterranean area during the Holocene, Territorio storico ed ambiente 3 . a cura di Claude Albore Livadie e Franco Ortolani, Edizioni Edipuglia, Centro Universitario Europeo per i Beni Culturali, Ravello, 461-475.

SAINTOT S., \& PASTY J.-F, avec la collaboration de ALIX Ph., BALLUT C., FONTANA L., GEORION C., MACABEO G,, TREFFORT J.-M., VERNET G., \& WATTEZ J., 2004 - Taphonomie et fonction des occupations pré et protohistorique du site Les Patureaux à Clermont-Ferrand, Puy-de-Dôme. Préhistoire du sud-ouest 2004-supplément $n^{\circ 9}$ : Sèmes rencontres méridionales de préhistoire récente, Auvergne et Midi, Actualité de la re. cherche, Clermont-Ferrand, 2002, 43-84.

VERNET G., RAYNAL J.-P., MIALLIER D., PILlEYRE T., FAIN .I., SANZELLE S., \& MONTRET M., 1990 - La retombée de la Moutade, marqueur stratigraphique de l'Allerød en Limagne septentrionale (Massif central, France). Comptes-Rendus de l'Académie des Sciences, Paris, 310 (II), 1077-1082.

VERNET G., 1992 - Message du volcanisme régional dans les formations quaternaires de Limagne occidentale (Massif central français). Minéraux denses et retombées. Thèse de Doctorat, Université de Bordeaux 1, $\mathrm{n}^{\circ} \mathbf{7 2 4}, 335 \mathrm{p}$.
VERNET G., \& RAYNAL J..R., 1995 - Le téphra des Roches, marqueu du volcanisme contemporain de la fin du Magdalénien dans le Massif Central français. Comptes-Rendus de l'Acadénie des Sciences, Paris, 321 (Ia), 713-720.

VERNET G., RAYNAL J.P., \& VIVENT D., 1996 - Le téphra de la rue Sous-les-Vignes, marqueur en Limagne du volcanisme trachytique postghciaire de la Chane des Puys. Comples-Rendus de l'Acadénie des Sciences, Paris, 310(II), 325-331.

VERNET G., RAYNAL J.-P, FAIN J., MLALLIER D. MONTRET M., PILLEYRE T., \& SANZELLE S., 1998 Tephrostratigraphy of the last $160 \mathrm{Ka}$ in Western Limagne (France). Quatemary International, 47/48, 139-146

VERNET G., RAYNAL J.-P., LEFEVRE D. \& KIEFFER G, 1999 - Téphras distales dans les dépôts du Pléstocene moyen de Venosa. In Notarchirico, Un sito del Pletstocene medio iniziale nel bacino di Venosa. A cura di Marcello Piperno, Edizioni Osanna, 1999, museo «L. Pigorini », 207-233.

VERNET G., \& RAYNAL J..P., 2000a - Un cadre téphrostratigraphique réactualisé pour la préhistoire tardiglaciaire et holocène de Limagne (Massif central, France). Comptes Rendus de l'Académie des Sciences, Sciences de la Terre et des planètes, Paris, 330. 399-405.

VERNET G., \& RAYNAL J.-P, 2000b - Hommes et volcans en Basse-Auvergne préhistorique. In Nouvelles archéologiques, Du terrain au laboratoire... Revute d'Auvergne, 554-555,76-87.

VERNET G., \& RAYNAL J.-P., 2001a " Tephrostratigraphy of Li magne revisited. Implications for the Last Glacial and Holocene Prehistory. In E. Juvigné \& J.P. Raynal (dir.), Tephras, chronology, archaeology. Les dossiers de l'Archeo-Logis $n^{0} \mathbf{1}$, CDERAD éd., Goudet, juillet 2001, 111-116

VERNET G., RAYNAL J.P., KIEFFER G., CAMUS G. GUADELLI J.-L., VIVENT D, MIALLIER D., SANZELLE S., PILLEYRE T., FAIN J., \& MONTRET M., 2001b - L'éruption trachyticue du puy Chopine (Puy-de-Dôme, France) : Impacts proximaux et distaux et implications écoarchéologiques. In E. Juvigné \& J.-P. Raynal (dir.), Tephras, chronology, archaeology. Les dossiers de $1^{*}$ Archeo-Logis n"1, CDERAD éd., Goudet, juillet $2001,227-234$

VERNET G., \& RAYNAL J.-P, 2002 - Eruptions trachytiques de la Chaîne des Puys (France) et leur impact sur les environnements. In J.-P. Raynal, C. Albore-Livadie, \& M. Pipeno (dir), Hommes et volcans: de l'éruption à l'objet. XIVth Congress UISPP, Liège, Les Dossiers de l'Archéologie n ${ }^{0}$, CDERAD ed., Goudet, 2002 , $49-56$.

VERNET G., 2004 - Les éruptions holocènes de la Chaine des Puys et le peuplement mésolithique et néolithique ancien en Basse Aum vergne (Massif central, France). Préhistoire du sud-ouest 2004. supplement $n^{* 9}$, 5 èmes rencontres méridionales de préhistoire ré. cente, Auvergne et Midi, Actualité de la recherche, Clermont-Ferrand, 2002, 17-24.

VIVENT D., \& VERNET G., 2001 - Apport de la palynologie à l'étude des retombées volcaniques tardiglaciaires et holocènes de la Chaine des Puys enregistrées en Limagne (France). In E. Juvigné \& J.-P. Raynal (dir), Tephras, chronology, archaeology. Les dossiers de l'Archéo-Logis n 1 , CDERAD ed, Goudet, $255 \mathrm{~m} 262$.

WALKER G.P.L., 1971 - Grain size characteristics of pyroclastic deposits. Joumal of Geology, 79,696-714. 